\title{
Cancer Diagnostics Integrated With Therapeutics: A Comprehensive Approach to Managing the Disease
}

\author{
Hossein A. Ghanbari* \\ Panacea Pharmaceuticals, Inc, 207 Perry Parkway, Suite 2, Gaithersburg, MD 20877, USA
}

A promotional poster by the US Public Health Service in cooperation with the American Society for Control of Cancer dated 1938 hangs in our conference room and shows a rooster crowing at sunrise. The poster reads: "Early is the Watch Word for Cancer Control; early diagnosis/early treatment will save many lives; early cancer can be cured."

It is obvious that the prerequisite for early treatment is early diagnosis. Currently, it is soundly projected that early diagnosis increases the survival of cancer patients by five years, making it the best "treatment" alternative yet.

Currently, there are a number of in vitro (laboratory) as well as in vivo (imaging) cancer diagnostics procedures available. However, the specificity and sensitivity of many of these tests are barely acceptable, and the diagnosis of cancer is usually made with the aid of other parametersbiopsy being the most definitive. More important, and more relevant to this editorial, the diagnostic markers in many of these cancer tests may not have anything to do with cancer biology or etiology; they just happen to be there. The best and well-known example is the prostatespecific antigen (PSA) test for prostate cancer that measures PSA production (and hence its serum level) which is elevated in individuals with large prostate, enlarged prostate, or enlarging or inflamed prostate (by injury, infection, cancer, etc). Although patients with prostate cancer usually have elevated PSA levels, a majority of people with abnormally high serum PSA levels do not have prostate cancer.

On the treatment side, physicians generally consider cancers that affect each organ or group of organs as "distinct" diseases (eg, brain tumor, prostate cancer, lung cancer, colon cancer, breast cancer, ovarian cancer, etc). For physicians, particularly surgeons, caring for cancer patients, this perspective makes a lot of sense because each tumor presents itself differently and has different implications and ramifications for individual patients. These differences have a considerable impact on the strategy for patient management. However, in drug discovery process, one needs to be more open-minded and treat cancer as a biological phenomenon with a mechanism of action

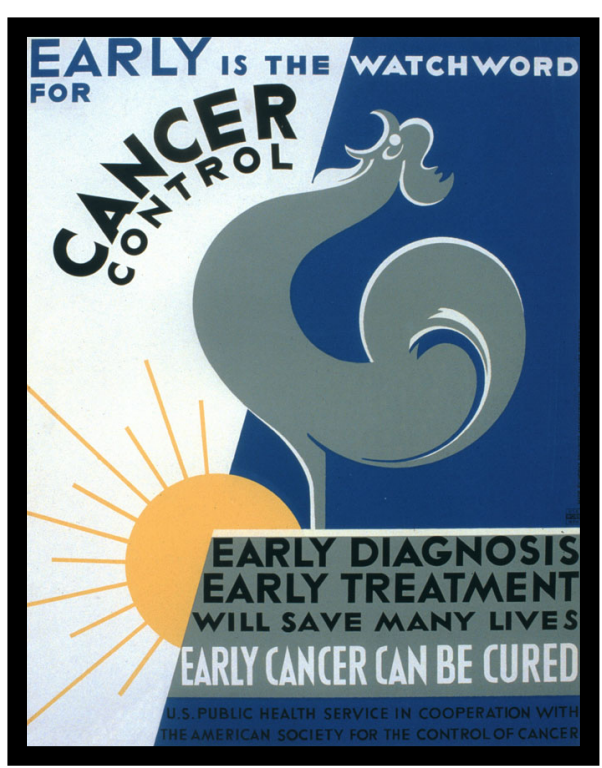

FIgURE 1. A promotional poster by the US Public Health Service in cooperation with the American Society for Control of Cancer dated 1938

spanning over various “cancers.” Drug discovery scientists need not be restrictive toward any particular cancer, and physicians need not be skeptical toward a pan approach to cancer diagnosis and treatment. Scientists and physicians alike need to actively consider prevention in their approach for patient management.

Ideally, the goal of cancer patient management is to tie the diagnosis with the treatment. We are working toward this goal by continuing research and development in which the diagnostic marker and therapeutic target is the very same molecule. It is certainly possible to measure serum and tissue levels of this molecule for diagnosis, to target it for treatment, to examine it for prognosis, and subsequently use it for surveillance, and even use it to predict the aggressiveness of cancer and choose the treatment option. 
The diagnostic marker and therapeutic target in our cancer discovery program is human aspartyl (asparaginyl) $\beta$-hydroxylase (HAAH). This enzyme is an $\alpha$ ketoglutarate-dependent deoxygenase that catalyzes posttranslational hydroxylation of $\beta$ carbons of aspartyl and asparaginyl residues present in epidermal-growth-factor (EGF)-like domains of certain proteins, including notch and notch homologues, which have known roles in oncogenesis and cellular functions that propagate the malignant phenotype. Experimental overexpression of HAAH causes malignant transformation, and inhibition of over-expression or inactivation of HAAH results in normalization of malignant phenotypes. HAAH overexpression correlates with motility of malignant neoplastic cells; as such, it has a functional role in cell migration or invasiveness-properties required for infiltrative and metastatic tumor growth.

As a diagnostic marker, HAAH is found to be overexpressed in virtually every tissue of every cancer specimen and absent in noncancer tissues (with the exception of placental trophoblast cells) tested by immunohistochemical (IHC) methods. Moreover, preliminary studies using sandwich ELISA for analysis of serum samples have resulted in an assay specificity of $97 \%$ and sensitivity of 95\%-100\% (depending on the sample set). Gene expression analysis (RT-PCR or qRT-PCR) also has demonstrated the overexpression of HAAH in virtually every cancer tissue type tested and in none of the noncancer specimens.

Beyond just a simple diagnostic marker, HAAH levels could be used to predict the aggressiveness of cancer in the patient and guide the physician in choosing an appropriate treatment option. In a recent study by Takashi Maeda, MD, et al, "Clinicopathological Correlates of Aspartyl (Asparaginyl) $\beta$-Hydroxylase OverExpression in Cholangiocarcinoma Cancer Detection and Prevention," it was demonstrated that HAAH expression in surgically resected intrahepatic cholangiocarcinoma significantly correlated with tumor size, growth types, differentiation, vascular invasion, and poor prognosis after surgery.

Further, HAAH has been proven, in principle, to be a valid target for cancer therapeutic development. Its relevance to cancer is well established by its biological role and its presence in most cancer cells. Upon overexpression, HAAH migrates to the cell surface and is primarily present in the infiltrating periphery of the tumor, making it an accessible target. Moreover, anti-HAAH antibodies (both naked and bound to toxins) internalize subsequent to binding to HAAH on the cell surface. Also, anti-HAAH antibodies and antisense inhibit proliferation, motility, and invasion in vitro and in vivo. Finally, in preliminary animal efficacy studies, injection of an anti-HAAH antibody has shown inhibition of growth and metastasis of established human colon cancer in a mouse xenograft model of human colon cancer metastasized to the liver.
Aside from HAAH's diagnostic and therapeutic value, based on the biological role of HAAH and the molecule, per se, it can be readily speculated that HAAH may be used as a target for the prevention of cancer. Obvious examples of preventive measures would be passive and active immunization for individuals at high risk. Again, the preventative approach can be keyed to HAAH levels, particularly for the period of post-treatment or post-surgery and other "at-risk" patients.

Many breakthroughs have been reported in the area of cancer diagnostics, therapeutics, and even prevention. Moreover, there has been a widespread tendency to combine these approaches. However, true integration of these approaches requires a common thread, and the discoverers and practitioners in oncology need to actively seek that common thread. Here, I have highlighted HAAH as a common thread to demonstrate that true integration of diagnostics and therapeutics is a possibility and its pursuit should stand at the forefront of efforts to manage and eventually tame cancer.

Hossein A. Ghanbari

Hossein A. Ghanbari is Chairman, $\mathrm{CEO} / \mathrm{CSO}$, and a cofounder of Panacea Pharmaceuticals; and Chairman of the Alzheimer's Corporation. Dr Ghanbari received his BS, with distinction, from the American University of Beirut (1970), his Master's degree (1974) and $\mathrm{PhD}$ (1975) in biochemistry from Pennsylvania State University. Dr Ghanbari worked at Abbott Laboratories, for ten years, where he developed several pharmaceutical and diagnostic products. One of his many notable accomplishments was the development of the first marketed Alzheimer's test. He was a member of the NDA Team that developed Leupron, a drug for metastatic prostate cancer. He served on Abbott's Technical Advisory Board and was inducted into the Volwiler Society reserved for the most accomplished scientists. Subsequently, he cofounded Molecular Geriatrics Corporation and served as its Senior Vice-President for R\&D. Dr Ghanbari served as Nymox Pharmaceutical's Senior Vice-President for R\&D and Strategic Planning, as well as Director. There he developed the first marketed CSF-based and urine-based Alzheimer's tests. Prior to his joining the industry sector, Dr Ghanbari worked in academia for seven years, mainly with Pennsylvania State University, AryaMehr University of Technology, and University of Florida. Aside from his scientific accomplishments, Dr Ghanbari is the inventor on more than 60 US and international patents/patent applications.

\footnotetext{
*E-mail: hag@panaceapharma.com
} 


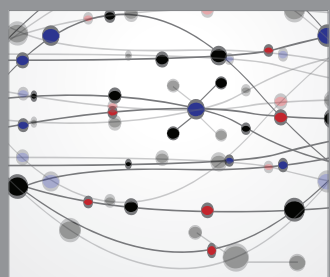

The Scientific World Journal
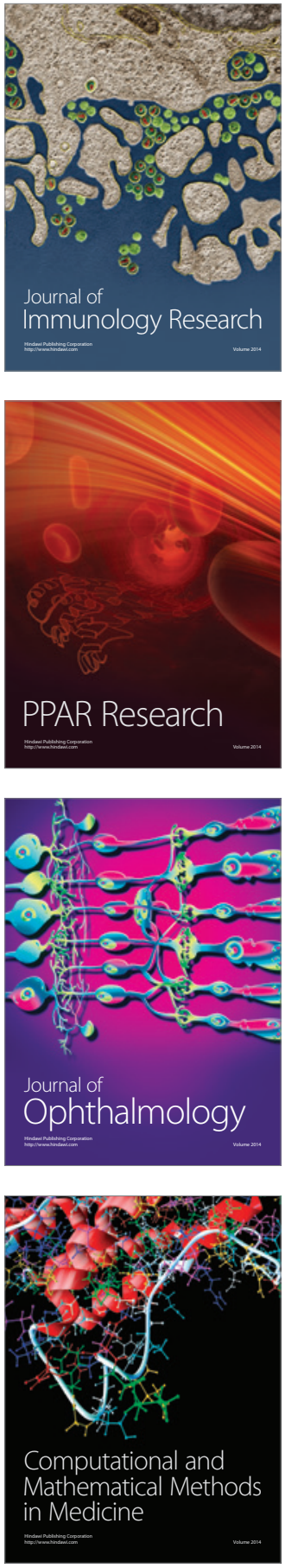

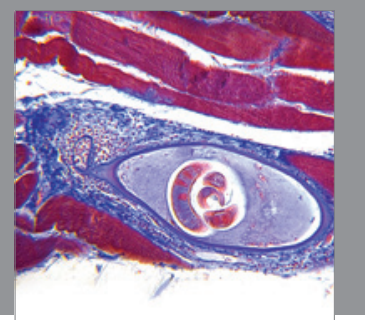

Gastroenterology

Research and Practice
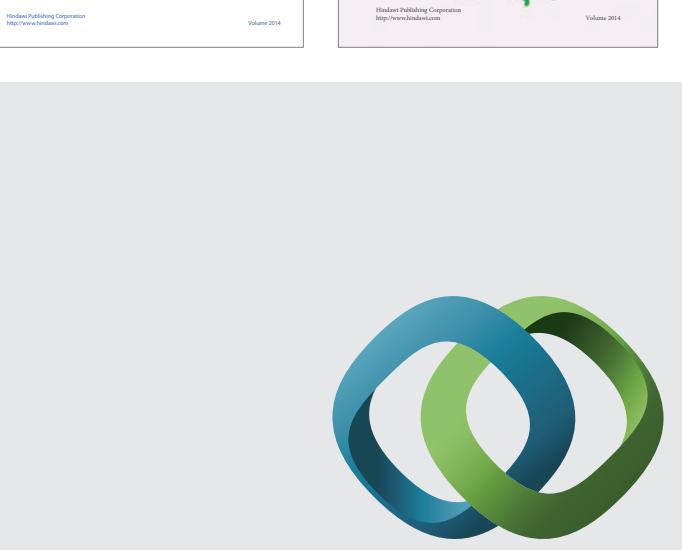

\section{Hindawi}

Submit your manuscripts at

http://www.hindawi.com
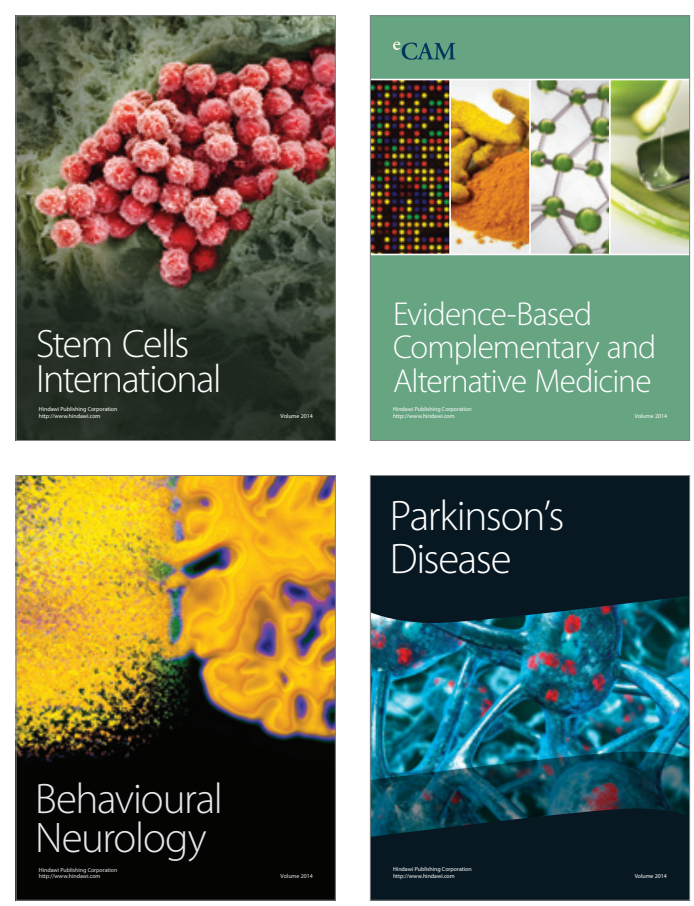

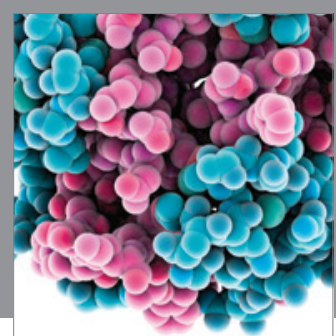

Journal of
Diabetes Research

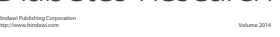

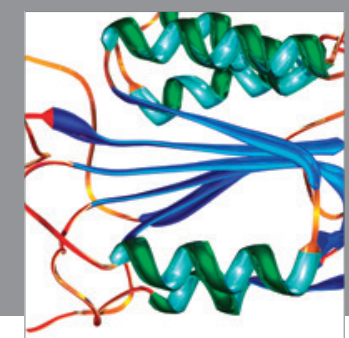

Disease Markers
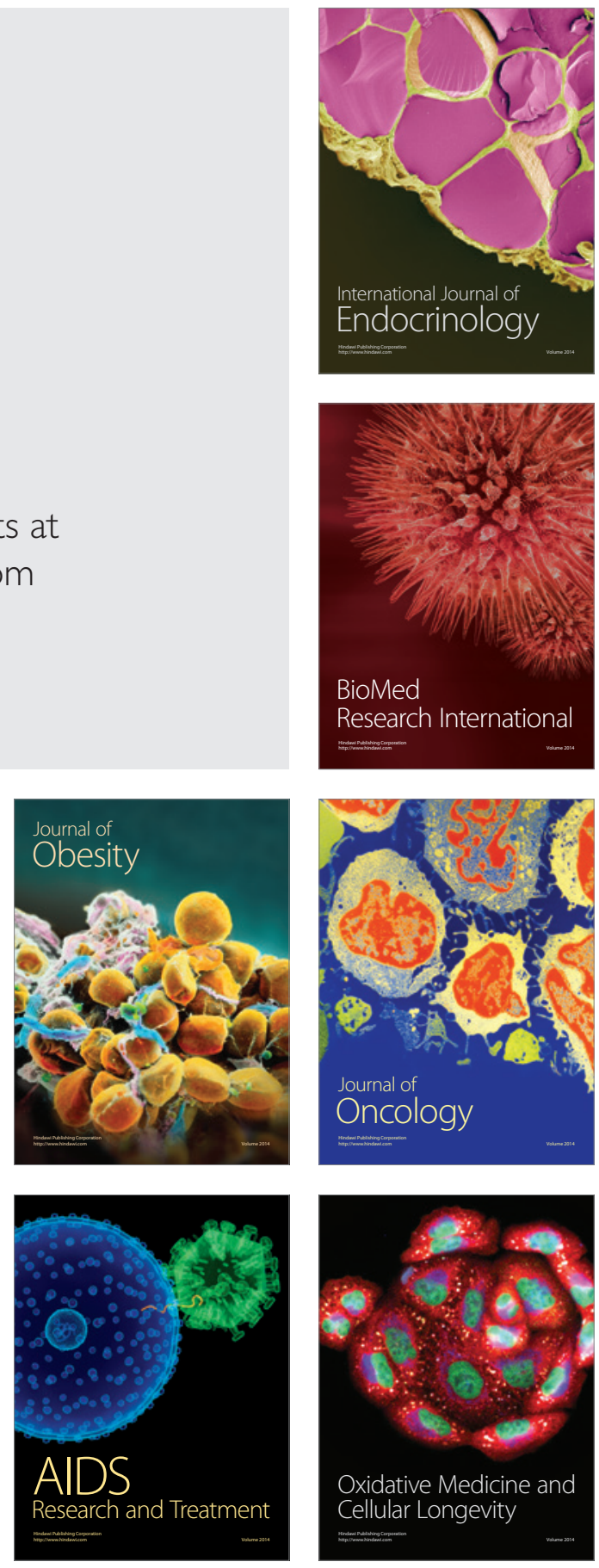